\title{
DINI-Wettbewerb um die besten Konzepte für hybride Lehr- und Lernformate
}

\section{Die Deutsche Initiative für Netzwerkinformation (DINI) sucht Ideen für innovative Lehr- und Lernkonzepte an Hochschulen und Universitäten}

https://doi.org/10.1515/bd-2022-0006

Zum Beginn der Corona-Pandemie haben Hochschulen und Universitäten Konzepte und Werkzeuge des rein digitalen Lehrens und Lernens schnell und erfolgreich umgesetzt. Doch auch wenn digitale Lern- und Lehrumgebungen viele Vorteile bringen, stellen sie den informellen Austausch mit Lehrenden und Studierenden bisher nur bedingt sicher. Der Mangel an sozialer Interaktion an Hochschulen und Universitäten ist in der Pandemie deutlich geworden.

Die Deutsche Initiative für Netzwerkinformation (DINI) sucht jetzt unter dem Motto „Neustart! Gemeinsam hybrides Lernen gestalten“ in einem studentischen Wettbewerb nach den besten Ideen für Lehr- und Lernkonzepte, die digitale Online-Distanzlehre und Präsenzlehre gemeinsam in den Blick nehmen.

Mit der Öffnung hin zur Präsenzlehre ist es nötig, innovative und kreative Ansätze hybrider Lehr- und Lernformate zu entwickeln und umzusetzen, die die Interaktionspotentiale zwischen Präsenzszenarien vor Ort und mobilen digitalen Szenarien neu denken. Hierbei ist zu berücksichtigen, dass diese hybride Interaktion nicht nur eine technisch-informationelle sondern auch eine soziale Komponente hat. Es ergeben sich aus der Nutzbarmachung solcher Konzepte nebenbei auch neue Chancen für ökologische Nachhaltigkeit, die internationale Vernetzung von Studierenden und Lehrenden sowie für Inklusion und soziale Teilhabe.

DINI-Vorsitzender Dr. Helge Steenweg sagt zum Start des Wettbewerbs: „Wir freuen uns über ideenreiche Impulse der Studierenden zur Weiterentwicklung von Konzepten für hybride Lehr- und Lernformate. Als Dienstleister für die Hochschullehre arbeiten wissenschaftliche Bibliotheken, Medieneinrichtungen und Rechenzentren ständig an der Weiterentwicklung ihrer Angebote. Der DINI-Wettbewerb sammelt Vorschläge zur Verbesserung der Lehre, prämiert die besten Ideen und fördert den Transfer der studentischen Visionen in die Praxis der wissenschaftlichen Einrichtungen in Deutschland.“

DINI sucht in dem Wettbewerb nach:

- innovativen Ideen und erprobten Lehr- und Lernformaten,

- studentischen Initiativen zum hybriden Lernen und zur Vernetzung, 
- kreativen Umsetzungen gleichzeitig virtueller und realer Lernumgebungen, die auf soziale Präsenz setzen,

- nachhaltigen Konzepten zum gemeinsamen Lernen in hybriden Settings,

- auf unterschiedliche Fachkontexte übertragbare Tools zum orts- und zeitunabhängigen Lernen,

- Tools und Skills für hybrides Lernen.

Bis zum 1. Juni 2022 können sich Studierende aller Hochschulen in Deutschland als Einzelpersonen oder Teams an dem Wettbewerb beteiligen. Die Visionen können als Ideenskizzen, Konzeptarbeiten oder andere kreative Beiträge in digitaler Form eingereicht werden. Kriterien des Wettbewerbs sind Innovation, Kreativität, Usability, Realisierbarkeit und Reichweite der eingereichten Beiträge. Die besten Ideen werden mit bis zu 5.000 Euro prämiert.

Mitglieder der Jury sind u. a.:

- Prof. Dr. Kerstin Mayrberger, Universität Hamburg, Professorin für Lehren und Lernen an der Hochschule mit dem Schwerpunkt Mediendidaktik,

- Prof. Dr. Gerhard Peter, Mitglied des DINI-Beirats und Professor für Informatik (emeritiert),

- Anne Prill, CHE Centrum für Hochschulentwicklung, Projektmanagerin des Hochschulforums Digitalisierung,

- Prof. Dr. Mandy Schiefner-Rohs, Technische Universität Kaiserslautern, Professorin für Allgemeine Pädagogik mit dem Schwerpunkt Schulentwicklung,

- Dr. Helge Steenweg, DINI-Vorsitzender und Direktor der Universitätsbibliothek Stuttgart.

Der DINI-Wettbewerb steht unter der Schirmherrschaft des Hochschulforum Digitalisierung. Der Wettbewerb für neuartige Lernkonzepte an Hochschulen und Universitäten sammelt zum sechsten Mal Ideen zur Verbesserung des digital unterstützten Lehrens und Lernens.

Weitere Informationen zum DINI-Wettbewerb: Neustart! Gemeinsam hybrides Lernen gestalten - Deutsche Initiative für Netzwerkinformation e.V. (dini.de): https://dini.de/wettbewerbe/neustart-gemeinsam-hybrides-lernen-gestalten.

\section{Über die Deutsche Initiative für Netzwerkinformation (DINI)}

Die Deutsche Initiative für Netzwerkinformation (DINI) e. V. ist der überregionale Zusammenschluss von wissenschaftlichen Bibliotheken, Medieneinrichtungen, Rechenzentren und Fachgesellschaften in Forschung und Lehre in Deutschland. DINI ist Partner von Hochschulen und außeruniversitären Forschungseinrichtungen bei der Weiterentwicklung der Informationsinfrastrukturen. Website: https:// dini.de. 